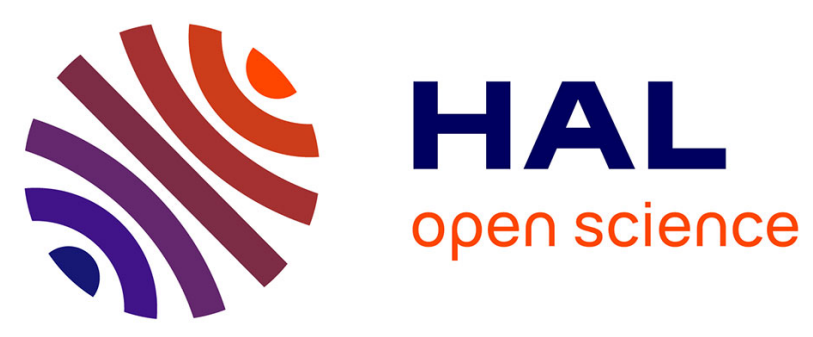

\title{
Minimum mean-square-error expression of LMMSE channel estimation in SISO OFDM systems
}

Vincent Savaux, Yves Louët, Moïse Djoko-Kouam, Alexandre Skrzypczak

\section{To cite this version:}

Vincent Savaux, Yves Louët, Moïse Djoko-Kouam, Alexandre Skrzypczak. Minimum mean-squareerror expression of LMMSE channel estimation in SISO OFDM systems. Electronics Letters, 2013, 49 (18), pp.1152-1154. 10.1049/el.2013.1993 . hal-00858869

\section{HAL Id: hal-00858869 \\ https://hal-centralesupelec.archives-ouvertes.fr/hal-00858869}

Submitted on 6 Sep 2013

HAL is a multi-disciplinary open access archive for the deposit and dissemination of scientific research documents, whether they are published or not. The documents may come from teaching and research institutions in France or abroad, or from public or private research centers.
L'archive ouverte pluridisciplinaire HAL, est destinée au dépôt et à la diffusion de documents scientifiques de niveau recherche, publiés ou non, émanant des établissements d'enseignement et de recherche français ou étrangers, des laboratoires publics ou privés. 


\section{Minimum mean-square-error expression of LMMSE channel estimation in SISO OFDM systems}

\author{
V. Savaux, Y. Louët, M. Djoko-Kouam and A. Skrzypczak
}

An expression of the minimum mean square error (MMSE) of the linear MMSE channel estimation is given in the case of a non-invertible channel covariance matrix, as in single-input single-output (SISO) OFDM system. A matrix expression, already proposed for a multiinput multi-output OFDM system in a previous article, is not valid in SISO. A new proof is then proposed, by deriving a scalar expression of the MMSE, which leads to solve an optimisation problem. Furthermore, we show that the proposed solution is the global minimum. Simulations validate the proposed development.

Introduction: Among the wide range of channel estimation techniques, the linear minimum mean square error (LMMSE) is known to be optimal in the sense of the MMSE criterion. In [1, 2], Biguesh and Gershman characterised the performance of LMMSE by expressing its MMSE. In the multi-input multi-output (MIMO) context described by Biguesh and Gershman [1, 2], the channel covariance matrix required for the LMMSE estimation is invertible. The expression of the MMSE is then derived by using the trace of the covariance matrix inverse.

In this Letter, we consider a single-input single-output (SISO) system. In this context, the channel covariance matrix is not invertible, so the MMSE of the LMMSE estimation given in $[1,2]$ has to be reconsidered, for it is not valid in SISO. Indeed, in SISO systems, the rank of the channel covariance matrix is equal to the length of the channel, which is lower than the fast Fourier transform (FFT) size. To get around this problem, we first develop a scalar expression of the mean square error, then we minimise this error under a given power constraint and we prove that the optimisation problem reaches the global minimum. We also show that, if the rank of the matrix tends to the FFT size, the proposed expression of MMSE matches the one given in [1, 2]. Thus, since the proposed expression can be used for SISO systems as well as for MIMO systems, it can be seen as a generalisation of the expression given in $[1,2]$.

The rest of this Letter is organised as follows: in the next two sections, we recall the expression of the LMMSE estimation and its MMSE according to the version of $[1,2]$. Thereafter, the following section gives a scalar expression of the mean square error and derives the MMSE for SISO systems, whose accuracy is confirmed by simulation. Finally, conclusions are drawn.

System model: We consider the transmission of OFDM symbols over a multipath channel. After the removal of the cyclic prefix and the discrete FT (DFT), we express the expression of the received OFDM symbol in the frequency domain as $\boldsymbol{U}=\underline{\boldsymbol{H}}+\boldsymbol{W}$, where $\boldsymbol{U}=\left[U_{0}, \ldots, U_{M-1}\right]^{\mathrm{T}}$,

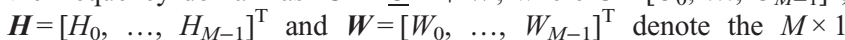
complex vectors of the received signal, the multipath channel and the Gaussian white noise, respectively. $M$ is the size of the DFT, which also tallies with the number of carriers per symbol in our model. The matrix $C$ is the $M \times M$ diagonal matrix of the transmitted signal containing the vector $\left[C_{0}, \ldots, C_{M-1}\right]$. Each $C_{m}$ is either a data element or a pilot, whose gain, phase and position are perfectly known at both transmitter and receiver sides. Let us assume a preamble scheme, i.e. one pilot symbol is dedicated to the channel estimation in the OFDM frame.

LMMSE channel estimation: From [3], we express $\hat{\boldsymbol{H}}^{\text {LMMSE }}$, the LMMSE estimation of the channel frequency response vector $\boldsymbol{H}=\left[H_{0}, H_{1}, \ldots, H_{M-1}\right]^{\mathrm{T}}$ performed by means of the preamble as

$$
\hat{\boldsymbol{H}}^{\mathrm{LMMSE}}=\underline{\boldsymbol{R}}_{H}\left(\underline{\boldsymbol{R}}_{H}+\sigma^{2}\left(\underline{\boldsymbol{C}}^{\mathrm{H}}\right)^{-1}\right)^{-1} \hat{\boldsymbol{H}}^{\mathrm{LS}}
$$

where $(.)^{\mathrm{H}}$ is the Hermitian transpose and $\sigma^{2}$ is the noise variance Whatever $m=0,1, \ldots, M-1$, the pilot subcarriers $C_{m}$ have a power $\mathcal{P}=C_{m} C_{m}^{*},(.)^{*}$ being the complex conjugate. The vector $\hat{\boldsymbol{H}}^{\mathrm{LS}}$ is the least-square (LS) channel estimation expressed by $\hat{\boldsymbol{H}}^{\mathrm{LS}}=\underline{\boldsymbol{C}}^{-1} \boldsymbol{U}=$ $\boldsymbol{H}+\boldsymbol{C}^{-1} \boldsymbol{W} . \boldsymbol{R}_{H}$ is the $M \times M$ channel frequency covariance matrix defined by $\underline{\boldsymbol{R}}_{H}=E\left\{\boldsymbol{H} \boldsymbol{H}^{\mathrm{H}}\right\}$, where $E\{$.$\} is the mathematical expectation.$ From [3] and considering that each multipath channel coefficient follows a zero-mean Gaussian process, we express the components $\left(\underline{\boldsymbol{R}}_{H}\right)_{u, v} u$ th row and $v$ th columns) of $\underline{\boldsymbol{R}}_{H}$ are the Fourier transform of the power intensity profile of the channel $\Gamma(\tau)$. The most common model for the channel intensity profile is the decreasing exponential [3] given by

$$
\Gamma(\tau)=\alpha \mathrm{e}^{\left((-\tau) /\left(\tau_{\max }\right)\right)} \quad \text { if } \tau \in\left[0, \tau_{\max }\right]
$$

and 0 elsewhere, $\alpha$ being a normalisation coefficient and $\tau_{\max }$ the maximum path delay. This is also the profile followed by the eigenvalues $\lambda_{0}, \lambda_{1}, \ldots, \lambda_{M-1}$ of $\underline{\boldsymbol{R}}_{H}$ when arranged in the decreasing order In this discrete formalism, we define $L$ as the length of the channel, i. e. the number of non-null eigenvalues of $\underline{\boldsymbol{R}}_{H}$. In SISO systems, the channel covariance matrix is then of rank $L$ [3]. Fig. 1 illustrates the shape of $\lambda_{0}, \lambda_{1}, \ldots, \lambda_{M-1}$, or in an equivalent way, the shape of $\Gamma(\tau)$ for $M=128$ and $L=32$. Furthermore, the channel is supposed to be normalised, i.e. $(1 / M) \sum_{m=0}^{M-1} \lambda_{m}=1$.

We now express the MMSE of the LMMSE channel estimation given in $[1,2]$. We remind that this expression is valid for full rank channel covariance matrices, i.e. when a well-sized MIMO system is considered

$$
\operatorname{MMSE}_{\mathrm{LMMSE}}=\frac{M \sigma^{2}}{M \mathcal{P}+\sigma^{2} \operatorname{tr}\left(\underline{\boldsymbol{R}}_{H}^{-1}\right)}
$$

where $\operatorname{tr}($.$) is the trace application. From (2), we note that the inverse$ covariance matrix $\underline{\boldsymbol{R}}_{H}^{-1}$ is required to express the minimum mean square error of the LMMSE channel estimation. In SISO systems, the covariance matrix $\underline{\boldsymbol{R}}_{H}$ has rank $L$ such as $L<M$, as depicted in Fig. 1 . As a consequence, $\boldsymbol{R}_{H}$ is not invertible. This observation motivates this Letter. Indeed, in the next section, we propose a new expression of the MMSE $E_{\text {LMMSE }}$ which does not require the invert of $\underline{\boldsymbol{R}}_{H}$, as the usual expression (2) does.

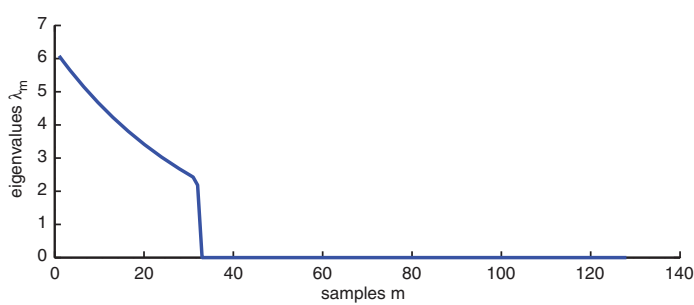

Fig. 1 Profile of eigenvalues $\lambda_{m}$ of the channel, for $M=128$ and $L=32$

Expression of the MMSE of LMMSE channel estimation in SISO systems: In this section, we first propose a scalar expression of the mean-square-error function $J_{\text {LMMSE }}$ from (1). Second, we minimise the error function subject to the pilot power constraint. From (1), we derive the mean-square-error function $J_{\mathrm{LMMSE}}$ :

$$
\begin{aligned}
J_{\text {LMMSE }}= & \frac{1}{M} \operatorname{tr}\left(E\left\{\left(\boldsymbol{H}-\hat{\boldsymbol{H}}^{\mathrm{LMMSE}}\right) \boldsymbol{H}^{\mathrm{H}}\right\}\right. \\
& \left.-E\left\{\left(\boldsymbol{H}-\hat{\boldsymbol{H}}^{\mathrm{LMMSE}}\right)\left(\hat{\boldsymbol{H}}^{\mathrm{LMMSE}}\right)^{\mathrm{H}}\right\}\right)
\end{aligned}
$$

Using the orthogonality principle [4], the second term of the right part of (3) is equal to zero. Since $\boldsymbol{H}$ and $\boldsymbol{W}$ are uncorrelated zero-mean processes, we have $E\left\{\boldsymbol{H} \boldsymbol{W}^{\mathrm{H}}=0\right\}$. The development of (3) then yields

$$
J_{\mathrm{LMMSE}}=\frac{1}{M} \operatorname{tr}\left(\underline{\boldsymbol{R}}_{H}\left(\frac{1}{\sigma^{2}} \underline{\boldsymbol{R}}_{H}\left(\underline{\boldsymbol{C}}^{\mathrm{H}}\right)+\underline{\boldsymbol{I}}\right)^{-1}\right)
$$

(5) becomes (4) where $\underline{\boldsymbol{I}}$ is the identity matrix. Since $\underline{\boldsymbol{R}}_{H}$ is a Hermitian and positive definite matrix, and $\left(\underline{C C}^{\mathrm{H}}\right)$ is a multiple of the identity matrix, $\left(1 /\left(\sigma^{2}\right)\right) \underline{\boldsymbol{R}}_{H}\left(\underline{\boldsymbol{C}}^{\mathrm{H}}\right)+\underline{\boldsymbol{I}}$ is also a Hermitian and positive definite matrix having the same basis of eigendecomposition as $\underline{\boldsymbol{R}}_{H}$. We can then write $\underline{\boldsymbol{R}}_{H}=\underline{\boldsymbol{Q}}_{H} \underline{\boldsymbol{Q}}^{\mathrm{H}}$ and $\left(1 /\left(\sigma^{2}\right)\right) \underline{\boldsymbol{R}}_{H}\left(\underline{\boldsymbol{C}}^{\mathrm{H}}\right)+\underline{\boldsymbol{I}}=\underline{\boldsymbol{Q}} \underline{\boldsymbol{D}}^{\mathrm{H}}$, where $\underline{\boldsymbol{Q}}$ is a unitary matrix. The $m$ th components of $\underline{\boldsymbol{D}}_{H}$ and $\underline{\overline{\boldsymbol{D}}}$ are $\lambda_{m}$ and $\left(\left(\left(C_{m} C_{m}^{*} \lambda_{m}\right) /\left(\sigma^{2}\right)\right)+1\right)$, respectively. By means of the eigendecomposition and remembering that $\lambda_{m}=0$ for $m=L, L+1, \ldots, M-1$, we finally obtain the scalar expression of the error function

$$
J_{\text {LMMSE }}=\frac{1}{M} \sum_{m=0}^{L-1} \frac{\lambda_{m} \sigma^{2}}{\lambda_{m} C_{m} C_{m}^{*}+\sigma^{2}}
$$


(6) becomes (5) We now solve the minimising problem

$$
\min _{C_{m}^{*}}\left(J_{\text {LMMSE }}\right), \quad \text { subject to } \mathcal{P}=\frac{1}{L} \sum_{m=0}^{L-1} C_{m} C_{m}^{*}
$$

To this end, we use the Lagrange multiplier method so that we can write the Lagrangian function, associated with the minimisation problem, as follows:

$$
\mathcal{L}=\frac{1}{M} \sum_{m=0}^{L-1} \frac{\lambda_{m} \sigma^{2}}{\lambda_{m} C_{m} C_{m}^{*}+\sigma^{2}}+\mu\left(\frac{1}{L} \sum_{m=0}^{L-1} C_{m} C_{m}^{*}-\mathcal{P}\right)
$$

(7) becomes (6) where $\mu$ is the Langrange multiplier. Whatever $m=0,1$, ... $L-1$, the partial derivatives of the Lagrangian $\mathcal{L}$ must satisfy $\partial \mathcal{L} / \partial C_{m}^{*}=0$ so we deduce that $C_{m} C_{m}^{*}=\sqrt{\left(\sigma^{2} L\right) / \mu M}-\sigma^{2} / \lambda_{m}$, and using the constraint $(1 / L) \sum_{m=0}^{L-1} C_{m} C_{m}^{*}=\mathcal{P}$, we obtain

$$
\sqrt{\frac{\sigma^{2} L}{\mu M}}=\mathcal{P}+\frac{1}{L} \sum_{m=0}^{L-1} \frac{\sigma^{2}}{\lambda_{m}}
$$

and then

$$
C_{m} C_{m}^{*}=\mathcal{P}+\frac{1}{L} \sum_{m^{\prime}=0}^{L-1} \frac{\sigma^{2}}{\lambda_{m^{\prime}}}-\frac{\sigma^{2}}{\lambda_{m}}
$$

(9) becomes (7). Inserting (7) into (5), we finally obtain the expression of the MMSE of the LMMSE channel estimation

$$
\operatorname{MMSE}_{\mathrm{LMMSE}}=\frac{1}{M} \frac{L^{2} \sigma^{2}}{L \mathcal{P}+\sum_{m=0}^{L-1}\left(\left(\sigma^{2}\right) /\left(\lambda_{m}\right)\right)}
$$

(10) becomes (8). We note that if $L=M$, i.e. if $\underline{\boldsymbol{R}}_{H}$ is full rank, (8) is then exactly equal to (2). The proposed expression of the MMSE of the LMMSE channel estimation (8) is then a generalisation of the one given in $[1,2]$. However, (8) may lead to a local minimum, so let us investigate the error convexity.

Convexity of $J_{L M M S E}$ : We now give a sufficient condition on the eigenvalues $\lambda_{0}, \lambda_{1}, \ldots, \lambda_{L-1}$ so that ( 8$)$ is a global minimum. The minimum MMSE $_{\mathrm{LMMSE}}$ is global if $J_{\mathrm{LMMSE}}$ is convex. Since a sum of convex functions remains convex, whatever $m=0,1, \ldots, L-1$, let us define the extracted function $f$ as $f\left(C_{m}^{*}\right)=\left(\lambda_{m} \sigma^{2}\right) /\left(M\left(\lambda_{m} C_{m} C_{m}^{*}+\sigma^{2}\right)\right)$. It is known that $f$ is convex if $\operatorname{Re}\left\{1+\left(\left(C_{m}^{*} f^{\prime \prime}\left(C_{m}^{*}\right)\right) /\left(f^{\prime}\left(C_{m}^{*}\right)\right)\right)\right\}>0$. After some developments, and remembering that $M, \lambda_{m}$ and $\sigma^{2}$ are positive and real-valued, we obtain

$$
\frac{M\left(\lambda_{m} C_{m} C_{m}^{*}+\sigma^{2}\right)-2 \lambda_{m} C_{m} C_{m}^{*}}{M\left(\lambda_{m} C_{m} C_{m}^{*}+\sigma^{2}\right)}>0
$$

Since $M$ is the FFT size, we can reasonably assume $M>2$. Thus, the previous inequality is valid whatever the $\lambda_{m}$ values, and then $J_{\text {LMMSE }}$ is convex. Consequently, this proves that (8) is a global minimum of the error function.

Validation of the theoretical results: Fig. 2 displays the MMSE of LMMSE channel estimation against $\mathcal{P} / \sigma^{2}$, and compares the theoretical expression (8) to simulation. We remind that the channel is the one described by its eigenvalues in Fig. 1, i.e. considering a decreasing exponential intensity profile and with a length $L=32$. The pilot power is normalised, that is $\mathcal{P}=1$. Furthermore, we compare the MMSE of LMMSE with the MMSE of LS, given by $\mathrm{MMSE}_{\mathrm{LS}}=\sigma^{2} / \mathcal{P}$.

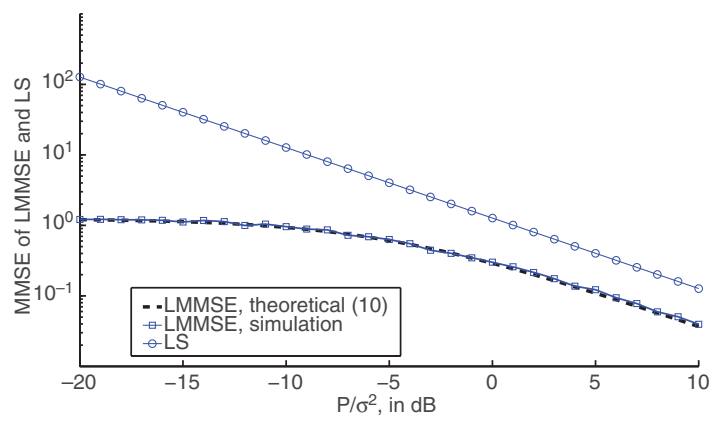

Fig. 2 MMSE of LMMSE and LS estimations against $\mathcal{P} / \sigma^{2}$

We observe that the curve of MMSE of LMMSE drawn by simulation exactly matches the theoretical one. It then validates the expression of MMSE $_{\mathrm{LMMSE}}$ given in (8). Furthermore, by comparing LMMSE and LS estimations, we retrieve the properties given in $[1,2]$, i.e. $\mathrm{MMSE}_{\mathrm{LMMSE}}<\mathrm{MMSE}_{\mathrm{LS}}$ whatever the value of $\sigma^{2}$

Conclusion: In this Letter, we proposed an MMSE expression of the LMMSE estimation which is a generalisation of the one given in $[1,2]$ for non-invertible channel covariance matrices. To this end, we give a scalar expression of the mean square error, we derive the MMSE by considering an optimisation problem under constraint, and we proved that this minimisation is global. Simulations finally validate the analytical expression that we derived.

(C) The Institution of Engineering and Technology 2013

21 June 2013

doi: 10.1049/el.2013.1993

One or more of the Figures in this Letter are available in colour online.

V. Savaux (Supélec, Rennes, France. Also with ECAM-Rennes, Rennes, France)

E-mail: vincent.savaux@ecam-rennes.com

Y. Louët (Supélec, Rennes, France)

M. Djoko-Kouam (ECAM-Rennes, Rennes, France)

A. Skrzypczak (Zodiac Aerospace, Caen, France)

\section{References}

1 Biguesh, M., and Gershman, A.B.: 'Downlink channel estimation in cellular systems with antenna arrays at base stations using channel probing with feedback', EURASIP J. Appl. Signal Process., 2004, 9, pp. 1330-1339

2 Biguesh, M., and Gershman, A.B.: 'Training-based MIMO channel estimation: a study of estimator tradeoffs and optimal training signals', IEEE Trans. Signal Process., 2006, 54, pp. 884-893

3 Edfors, O., Sandell, M., van de Beek, J.-J., Wilson, S.K., and Börjesson, P.O.: 'OFDM channel estimation by singular value decomposition', IEEE Trans.Commun., 1998, 46, pp. 931-939

4 Kay, S.M.: 'Fundamentals of Statistical Signal Processing: Estimation Theory' (Prentice-Hall, 2003) 